\title{
Flavourful SMEFT likelihood for Higgs and electroweak data
}

\author{
Adam Falkowski ${ }^{a}$ and David Straub ${ }^{b}$ \\ ${ }^{a}$ Université Paris-Saclay, CNRS/IN2P3, IJCLab, \\ Orsay 91405, France \\ ${ }^{b}$ Excellence Cluster Universe, \\ Boltzmannstr. 2, Garching 85748, Germany \\ E-mail: adam.falkowski@th.u-psud.fr, straub@protonmail.com
}

ABSTRACT: We perform an updated fit to LHC Higgs data and LEP electroweak precision tests in the framework of the Standard Model Effective Field Theory (SMEFT). We assume a generic structure of the SMEFT operators without imposing any flavour symmetries. The implementation is released as part of the public global SMEFT likelihood. This allows one to fit parameters of a broad class of new physics models to combined Higgs, electroweak, quark flavour, and lepton flavour observables.

Keywords: Beyond Standard Model, Effective Field Theories, Higgs Physics

ARXIV EPRINT: 1911.07866 


\section{Contents}

1 Introduction 1

2 SMEFT framework 3

3 Experimental data and fit methodology 3

4 Confidence intervals for Wilson coefficients 5

5 Applications $\quad 9$

5.1 Oblique parameters 9

$\begin{array}{lll}5.2 & \text { Custodial vector resonance model } & 10\end{array}$

6 Summary 11

\section{Introduction}

After the discovery of the $125 \mathrm{GeV}$ boson $[1,2]$, the Higgs searches at the LHC have turned into precision tests of the Standard Model (SM). The SM predicts the coupling strength between each fundamental particle and the Higgs in terms of that particle's mass, without any adjustable parameters. Beyond the SM (BSM), however, these couplings can be modified, therefore measurements of Higgs production cross sections and decay rates may reveal the fundamental theory underlying the SM. It is beneficial to describe Higgs coupling modifications in the model-independent language of effective field theory (EFT) [3-5]. Assuming new physics decouples, that is to say the masses of non-SM particles are parametrically larger than the weak scale, the relevant effective theory at energies $E \sim$ $m_{h} \approx 125 \mathrm{GeV}$ is the so-called SMEFT. That theory has the same particle content and local symmetry as the SM, but it admits interaction terms (operators) in the Lagrangian with canonical dimensions larger than four. The Higgs data can be interpreted as constraints on Wilson coefficients of the higher-dimensional operators.

Advantages of the SMEFT are not restricted to LHC Higgs physics. The theory offers a universal framework to describe a vast spectrum of precision measurements performed in weak scale colliders such as the LHC, Tevatron, or LEP. In particular, it is a perfect language to describe the so-called electroweak precision tests, that is accurate measurements of $Z$ and $W$ boson properties. A straightforward observation is that SMEFT operators may simultaneously affect both Higgs and electroweak observables. Therefore it makes sense to combine the two sets in order to increase the constraining power of data [6-16]. This work represents another step forward in this direction. 
In this paper we construct a likelihood for Wilson coefficients of dimension-6 SMEFT operators using a large set of Higgs and electroweak observables. Compared to previous analyses, the main novelties are:

1. We do not impose any simplifying assumptions about the flavour structure of dimension-6 operators. This makes the analysis considerably more difficult, but the advantage is that our results are more general. The likelihood we provide can thus be used to constrain a variety of BSM models [17] beyond the $\mathrm{U}(3)^{5}$ or minimal flavour violation paradigm. In particular, it can be applied to models addressing the so-called B-meson anomalies. Indeed, these models aim at addressing the deviations from the SM predictions in the measured values of $R_{K^{(*)}}[18,19]$ and $R_{D^{(*)}}$ [20]. By the very definition of these observables, any explanation must involve new physics coupling with a different strength to the different lepton generations (and typically also to the different quark generations in order to satisfy phenomenological constraints). Integrating out such new physics leads to the SMEFT with a flavour non-universal structure of dimension- 6 operators, which could not be constrained by previous combined Higgs and electroweak fits due to their assumption of flavour universality.

2. All code employed for our analysis is open source. This allows the community to scrutinize, reproduce, and modify our analysis, e.g. when new data becomes available. We follow the standard Wilson coefficient exchange format (WCxf) [21], such that our results can be easily imported by other analysis codes.

3. We include the most recent Higgs signal strength combinations from ATLAS and CMS based on up to $80 \mathrm{fb}^{-1}$ of the Run-2 data $[22,23]$.

Simultaneously to interpretations of high-energy collider data, much progress has been made on the front of low-energy observables measured at energies well below the weak scale. In refs. $[24,25]$ a treasure trove of such data was recast as constraints on the flavour-generic SMEFT. Moreover, a public code smelli ${ }^{1}$ providing a likelihood function in SMEFT Wilson coefficient space was released [26], based on the flavio observable calculator [27] and the wilson tool for running and matching Wilson coefficients [28]. Besides electroweak precision tests, the code includes information from quark flavour physics, lepton flavour physics, low-energy parity violation, and other low-energy precision tests. It is desirable to include the Higgs observables into the same framework, as many dimension-6 operators generated by typical BSM models are currently probed only via LHC Higgs searches. As a part of this work, the combined Higgs and electroweak likelihood was incorporated into the global SMEFT likelihood package smelli, which allows users to perform combined fits of BSM models to Higgs, electroweak, flavour, and other low-energy data.

This paper is organized as follows. In section 2 we briefly review the SMEFT framework in order to fix our conventions and notation. In section 3 we summarize the data used in this analysis and describe our methodology of constructing global likelihoods and parameter fitting. In section 4 we give the best fit and confidence intervals for the Wilson coefficients

\footnotetext{
${ }^{1}$ For details, see https://smelli.github.io.
} 
affecting the Higgs and/or electroweak observables at tree level. Section 5 contains two simple applications of our flavourful likelihood: one designed to connect and compare to previous work, and the other illustrating the relevance of working with a general flavour structure of dimension- 6 operators.

\section{SMEFT framework}

We briefly review the SMEFT framework to fix our notation. We consider the extension of the SM by all independent dimension-6 operators $Q_{i}$ invariant under the SM gauge symmetries $[29,30]$ :

$$
\mathcal{L}_{\mathrm{SMEFT}}=\mathcal{L}_{\mathrm{SM}}+\sum_{i} C_{i} Q_{i}
$$

We work in the Warsaw basis of operators [30], selecting the weak basis for fermions where the down-type quark and charged lepton mass matrices are diagonal (coinciding with the Warsaw basis as defined in the WCxf [21]). In our conventions the Wilson coefficients $C_{i}$ have dimensions $[\text { mass }]^{-2}$ and they count as $\mathcal{O}\left(\Lambda^{-2}\right)$ in the EFT expansion. Operators with dimensions higher than six, as well as dimension-5 operators (which only generate tiny neutrino masses and have no observable effect on Higgs or electroweak phenomenology) are ignored in this analysis. We also ignore CP-violating dimension- 6 operators. This is because the electroweak and Higgs observables we are concerned with in this paper are all CP-conserving. Therefore, the CP-violating operators affect our observables only at the quadratic level in the corresponding Wilson coefficients, thus at $\mathcal{O}\left(\Lambda^{-4}\right)$ in the EFT expansion. The electroweak parameters $g_{L}, g_{Y}, v$ are defined in the $\alpha-m_{Z}-G_{F}$ scheme. We take into account only tree-level effects of dimension- 6 operators, except in the observables where the SM contribution itself appears first at one loop. In this approximation, the operators affecting the Higgs and electroweak precision data are shown in table 1. The only four-fermion operator entering is the operator $\left[Q_{l l}\right]_{1221}$ that interferes with the SM amplitude in muon decay and thus modifies the relation between the muon lifetime (defining the Fermi constant $G_{F}$ ) and the electroweak parameters. ${ }^{2}$

\section{Experimental data and fit methodology}

We summarize the experimental data used in our analysis.

- The $Z$ and $W$ pole observables. These are largely unchanged compared to the analysis of ref. [31], see table 1 therein for the original references. The only new pieces of experimental information are i) the PDG combination for the $W$ mass [32], which includes the recent ATLAS measurement [33]; ii) the measurements of $\frac{\Gamma(W \rightarrow \tau \nu)}{\Gamma(W \rightarrow e \nu)}$ in D0 [34] and of $\frac{\Gamma(W \rightarrow \mu \nu)}{\Gamma(W \rightarrow e \nu)}$ in LHCb [35]; iii) the updated values of $\sigma_{\text {had }}^{0}$ and $\Gamma_{Z}$ following the recent revision of the LEP-1 integrated luminosity [36]. On the other hand, we

\footnotetext{
${ }^{2}$ The operators $\left[Q_{l l}\right]_{i j j i}$ and $\left[Q_{l l}\right]_{j i i j}$ are indistinguishable. In our conventions, only the ones with $i \leq j$ are included in the Lagrangian. Another convention encountered in the literature is that both are included and multiplied by the same Wilson coefficient, in which case our results for $\left[C_{l l}\right]_{1221}$ have to be multiplied by $1 / 2$.
} 


\begin{tabular}{|cccccc|}
\hline$Q_{\varphi D}$ & $\left(\varphi^{\dagger} D^{\mu} \varphi\right)^{*}\left(\varphi^{\dagger} D_{\mu} \varphi\right)$ & $Q_{\varphi l}^{(1)}$ & $\left(\varphi^{\dagger} i \stackrel{\leftrightarrow}{D}_{\mu} \varphi\right)\left(\bar{l} \gamma^{\mu} l\right)$ & $Q_{u \varphi}$ & $\left(\varphi^{\dagger} \varphi\right)(\bar{q} u \widetilde{\varphi})$ \\
$Q_{\varphi \square}$ & $\left(\varphi^{\dagger} \varphi\right) \square\left(\varphi^{\dagger} \varphi\right)$ & $Q_{\varphi l}^{(3)}$ & $\left(\varphi^{\dagger} i \stackrel{\leftrightarrow}{D}_{\mu}^{I} \varphi\right)\left(\bar{l} \tau^{I} \gamma^{\mu} l\right)$ & $Q_{d \varphi}$ & $\left(\varphi^{\dagger} \varphi\right)(\bar{q} d \varphi)$ \\
$Q_{\varphi B}$ & $\varphi^{\dagger} \varphi B_{\mu \nu} B^{\mu \nu}$ & $Q_{\varphi e}$ & $\left(\varphi^{\dagger} i \stackrel{\leftrightarrow}{D}_{\mu} \varphi\right)\left(\bar{e} \gamma^{\mu} e\right)$ & $Q_{e \varphi}$ & $\left(\varphi^{\dagger} \varphi\right)(\bar{l} e \varphi)$ \\
$Q_{\varphi W}$ & $\varphi^{\dagger} \varphi W_{\mu \nu}^{I} W^{I \mu \nu}$ & $Q_{\varphi q}^{(1)}$ & $\left(\varphi^{\dagger} i \stackrel{\leftrightarrow}{D}_{\mu} \varphi\right)\left(\bar{q} \gamma^{\mu} q\right)$ & $Q_{l l}$ & $\left(\bar{l} \gamma_{\mu} l\right)\left(\bar{l} \gamma^{\mu} l\right)$ \\
$Q_{\varphi W B}$ & $\varphi^{\dagger} \tau^{I} \varphi W_{\mu \nu}^{I} B^{\mu \nu}$ & $Q_{\varphi q}^{(3)}$ & $\left(\varphi^{\dagger} i \stackrel{\leftrightarrow}{D}_{\mu}^{I} \varphi\right)\left(\bar{q} \tau^{I} \gamma^{\mu} q\right)$ & $Q_{W}$ & $\epsilon^{I J K} W_{\mu}^{I \nu} W_{\nu}^{J \rho} W_{\rho}^{K \mu}$ \\
$Q_{\varphi G}$ & $\varphi^{\dagger} \varphi G_{\mu \nu}^{A} G^{A \mu \nu}$ & $Q_{\varphi u}$ & $\left(\varphi^{\dagger} i \stackrel{\leftrightarrow}{D} \mu\right)\left(\bar{u} \gamma^{\mu} u\right)$ & $Q_{G}$ & $f^{A B C} G_{\mu}^{A \nu} G_{\nu}^{B \rho} G_{\rho}^{C \mu}$ \\
\hline
\end{tabular}

Table 1. Dimension-6 SMEFT operators in the Warsaw basis relevant for the Higgs and electroweak observables at tree level.

no longer use the $V_{t b}$ determination from ref. [37] to constrain the $W t b$ vertex, as other dimension- 6 operators beyond those in table 1 may affect that measurement.

- The total and differential cross sections for $W W$ pair production measured in LEP-2 (tables 5.3 and 5.6 of ref. [38]). These are unchanged compared to the analysis of ref. [39].

- Combination of Run-1 ATLAS and CMS measurements of the Higgs signal strength measurements in 21 different channels (table 8 of ref. [40] with the correlation matrix in figure 27, plus the $\mu \mu$ signal strength). In addition, we use ATLAS [41] and CMS [42] Run-1 measurements of the signal strength in the $Z \gamma$ channel.

- Combined ATLAS measurement of the Higgs signal strength based on the $80 \mathrm{fb}^{-1}$ of run-2 data (table 6 of ref. [43] with the correlation matrix in figure 6). We also include the signal strength measurements in the $\mu \mu$ [44], $Z_{\gamma}$ [45], and $c \bar{c}$ [23] channels.

- Combined CMS measurement of the Higgs signal strength based on the $35.9 \mathrm{fb}^{-1}$ of run-2 data (table 3 of ref. [22] with the correlation matrix in the auxiliary material). We also include the signal strength measurements in the $Z_{\gamma}[46]$ and $c \bar{c}$ channels [47].

In order to take into account the correlations, we have to treat the experimental likelihoods as (multivariate) Gaussians. To this end, we symmetrize asymmetric uncertainties. Assuming Gaussian distributions for the LHC Higgs results does introduce into our analysis an additional uncertainty. That is however very difficult to quantify given the limited information shared by the LHC collaborations. This issue will be mitigated by future ATLAS/CMS combinations of the Higgs results, and hopefully resolved in the future when full likelihoods are released by the collaborations.

SMEFT corrections to electroweak observables are calculated analytically at tree level. For most of the Higgs observables they are determined numerically using the SMEFTsim model implementation [48] in Madgraph [49]. The exceptions are the $g g \rightarrow h$ and $h \rightarrow \gamma \gamma$ processes where we use analytic formulas, taking into account 1-loop effects due to modified 
Yukawa and $h V_{\mu} V_{\mu}$ couplings. Logarithmically enhanced one-loop corrections proportional to the dimension- 6 Wilson coefficient are included in our code via the renormalization group running effects [50]. Finite loop corrections (see e.g. [51-55]) other than those described above are ignored in our analysis. Our calculation of the Higgs and diboson observables include the effects due to modified $Z$ and $W$ couplings to fermions $[56,57]$. We take into account the interference effects in $h \rightarrow V^{(*)} V^{*} \rightarrow 4 f$ decays [58-60].

Having expressed all observables as linear functions of SMEFT Wilson coefficients, $\mathbf{O}_{\text {th }}\left(\mathbf{C}_{\mathbf{i}}\right)$, we construct the likelihood function $L\left(C_{i}\right)=e^{-\chi^{2}\left(C_{i}\right) / 2}$, with

$$
\chi^{2}\left(C_{i}\right)=\mathbf{x}\left(\mathbf{C}_{\mathbf{i}}\right)^{\mathbf{T}} \mathbf{S}^{-\mathbf{1}} \mathbf{x}\left(\mathbf{C}_{\mathbf{i}}\right),
$$

where $S$ is the experimental covariance matrix and

$$
\mathbf{x}\left(\mathbf{C}_{\mathbf{i}}\right)=\mathbf{O}_{\mathrm{th}}\left(\mathbf{C}_{\mathbf{i}}\right)-\mathbf{O}_{\exp }
$$

is the difference between the observables predicted in the SMEFT and measured experimentally. Given the precision of SM calculations of the observables in question, we can neglect all theory uncertainties and thus obtain a likelihood function that only depends on Wilson coefficients, and not on nuisance parameters.

\section{Confidence intervals for Wilson coefficients}

In this section we present marginalized $1 \sigma$ confidence intervals for the Wilson coefficients entering into our combined likelihood. This exercise illustrates, in a model-independent fashion, the constraining power of the current Higgs and electroweak data.

The electroweak and Higgs constraints dramatically differ in accuracy: for the former the typical precision is $\mathcal{O}(0.1 \%)$, while for the latter it is $\mathcal{O}(10 \%)$. In the Warsaw basis the two sets of constraints probe an overlapping set of Wilson coefficients, which leads to large correlations. For the sake of illustration, it is more transparent to work with certain linear combinations of Wilson coefficients, such that the strongly constrained combinations are isolated from the weakly constrained ones $[7,61]$. With this in mind, we define ${ }^{3}$ the following linear combinations of the Warsaw basis Wilson coefficients:

$$
\begin{aligned}
\delta g_{L}^{W \ell} & =C_{\varphi l}^{(3)}+f(1 / 2,0)-f(-1 / 2,-1), \\
\delta g_{L}^{Z \ell} & =-\frac{1}{2} C_{\varphi l}^{(3)}-\frac{1}{2} C_{\varphi l}^{(1)}+f(-1 / 2,-1), \\
\delta g_{R}^{Z \ell} & =-\frac{1}{2} C_{\varphi \ell}^{(1)}+f(0,-1) \\
\delta g_{L}^{Z u} & =\frac{1}{2} C_{\varphi q}^{(3)}-\frac{1}{2} C_{\varphi q}^{(1)}+f(1 / 2,2 / 3), \\
\delta g_{L}^{Z d} & =-\frac{1}{2} C_{\varphi q}^{(3)}-\frac{1}{2} C_{\varphi q}^{(1)}+f(-1 / 2,-1 / 3),
\end{aligned}
$$

\footnotetext{
${ }^{3}$ Note that our $\delta g$ 's differ by $1 / v^{2}$ from the ones defined in refs. [25, 31].
} 


$$
\begin{aligned}
\delta g_{R}^{Z u} & =-\frac{1}{2} C_{\varphi u}+f(0,2 / 3) \\
\delta g_{R}^{Z d} & =-\frac{1}{2} C_{\varphi d}+f(0,-1 / 3)
\end{aligned}
$$

where

$$
f\left(T^{3}, Q\right) \equiv\left\{-Q \frac{g_{L} g_{Y}}{g_{L}^{2}-g_{Y}^{2}} C_{\varphi W B}-\mathbf{1}\left(\frac{1}{4} C_{\varphi D}+\frac{1}{2} \Delta_{G_{F}}\right)\left(T^{3}+Q \frac{g_{Y}^{2}}{g_{L}^{2}-g_{Y}^{2}}\right)\right\} \mathbf{1},
$$

and $\Delta_{G_{F}}=\left[C_{H l}^{(3)}\right]_{11}+\left[C_{H l}^{(3)}\right]_{22}-\frac{1}{2}\left[C_{l l}\right]_{1221}$. In fact, $v^{2} \delta g^{V f}$ are the vertex corrections parametrizing deviations from the SM prediction of the $V=Z, W$ boson couplings to fermion $f$. Since they are probed by the LEP electroweak precision tests, almost all $\delta g$ 's are independently constrained with a good accuracy, except for one weakly constrained direction in the space of the light quark vertex corrections [31].

We also define another set of linear combinations of the Warsaw basis Wilson coefficients

$$
\begin{aligned}
\delta c_{z} & =C_{\varphi \square}-\frac{1}{4} C_{\varphi D}-\frac{3}{2} \Delta_{G_{F}}, \\
c_{z \square} & =\frac{1}{2 g_{L}^{2}}\left(C_{\varphi D}+2 \Delta_{G_{F}}\right), \\
c_{g g} & =\frac{4}{g_{s}^{2}} C_{\varphi G}, \\
c_{\gamma \gamma} & =4\left(\frac{1}{g_{L}^{2}} C_{\varphi W}+\frac{1}{g_{Y}^{2}} C_{\varphi B}-\frac{1}{g_{L} g_{Y}} C_{\varphi W B}\right), \\
c_{z z} & =4\left(\frac{g_{L}^{2} C_{\varphi W}+g_{Y}^{2} C_{\varphi B}+g_{L} g_{Y} C_{\varphi W B}}{\left(g_{L}^{2}+g_{Y}^{2}\right)^{2}}\right), \\
c_{z \gamma} & =4\left(\frac{C_{\varphi W}-C_{\varphi B}-\frac{g_{L}^{2}-g_{Y}^{2}}{2 g_{L} g_{Y}} C_{\varphi W B}}{g_{L}^{2}+g_{Y}^{2}}\right) .
\end{aligned}
$$

The important point is that, unlike $\delta g$, the combinations $c_{i}$ defined above do not affect electroweak observables at tree level. Therefore they are only loosely constrained by the less precise Higgs data, and they are weakly correlated with $\delta g$ 's.

We now present the best fit for the Wilson coefficients after a couple of simplifying but physically reasonable assumptions. First, we ignore the dependence of the likelihood on the Wilson coefficients affecting only the Yukawa couplings of the light fermions: $\left[C_{u \varphi}\right]_{i i}$, $\left[C_{d \varphi}\right]_{i i}, i=1,2$, and $\left[C_{e \varphi}\right]_{11}$. Currently, these are probed mostly via their contributions to the total Higgs width (thus affecting all observed branching fractions uniformly), and their effect is negligible unless the shift of the Yukawa couplings exceeds the SM Yukawa by orders of magnitude. Leaving these parameters in the fit would lead to flat directions. We also ignore the contributions to Higgs observables proportional to the Wilson coefficient $C_{G}$, which enters into our likelihood via its contributions to the $t \bar{t} h$ production. Leaving $C_{G}$ in the fit would result in a flat direction, approximately along the $C_{G} \approx\left[C_{u \phi}\right]_{33}$ line. 
One can invoke a theoretical argument in favour of dropping $C_{G}$ from the current analysis. The existing measurements of the $t$ th signal strength are sensitive to $\left|C_{G}\right| \sim \frac{1}{(600 \mathrm{GeV})^{2}}$. On the other hand, if the SMEFT describes a perturbative UV complete model then $O_{G}$ can be generated only at one loop level and we expect $\left|C_{G}\right| \ll \frac{1}{(600 \mathrm{GeV})^{2}}$ if the EFT description is valid. We also note that the $C_{G}$ parameter is much better probed by dijet observables [62], although a more careful analysis is needed to demonstrate that all flat directions are lifted in a combined electroweak+Higgs+dijet fit.

With the above assumptions, 31 independent combinations of Wilson coefficients are left as free parameters in the fit. Our results are shown in table 2. As advertised, the combinations $\delta g$ are more strongly constrained, at the level $|\delta g| \lesssim 10^{-2}-10^{-3}$, compared to the other combinations probed only by Higgs observables. There are a few exceptions from this rule, however. First, the uncertainty for the combinations $\delta g^{Z q}$ corresponding to light quark vertex corrections are larger [31]. While LEP-1 measures the total hadronic width with a per mille precision, it does not resolve all light quark couplings to $Z$ independently, leading to an approximate flat direction that is only lifted by less precise measurements. Second, some of the Wilson coefficients affecting Higgs observables only are strongly constrained when they compete with the SM loop-induced processes $\left(c_{g g}, c_{\gamma \gamma}\right)$, or with small SM Yukawa couplings $\left(\left[C_{e \varphi}\right]_{22},\left[C_{e \varphi}\right]_{33},\left[C_{d \varphi}\right]_{33}\right)$.

The best fit point has $\Delta \chi^{2}=\chi_{\mathrm{SM}}^{2}-\chi_{\min }^{2} \approx 36.7$, which translates to a p-value of approximately $20 \%$ for the SM hypothesis. There is no significant hint of physics beyond the SM in the fit, even though some of the individual couplings in table 2 display pulls (defined simply as the number of Gaussian standard deviations away from 0 ) of order $3 \sigma$.

The correlation matrix is shown in figure 1. The change of variables in eq. (4.1) and eq. (4.3) disentangles most of the large correlations present if the original Warsaw basis variables. Some $\mathcal{O}(1)$ correlations remain, however. Notably, there are large correlations between the combinations $c_{z z}$ and $c_{z}$. This is due to the fact that the current Higgs data poorly disentangle different possible Lorentz structures of the Higgs couplings to electroweak gauge bosons. This situation can be somewhat improved by including in the analysis, in addition to the signal strength, transverse-momentum distributions in the gluon fusion or invariant mass distributions in the associated Higgs production [57, 63-65]. We note that, for strongly correlated Wilson coefficients, the best fit values and the magnitude of the errors may be sensitive to including in the observables quadratic $\left(\mathcal{O}\left(\Lambda^{-4}\right)\right)$ effects in Wilson coefficients. Indeed, for $c_{z z}$ and $c_{z}$ we find that the error change by $\sim 50 \%$ upon including the quadratic corrections, with smaller or negligible effects for other Wilson coefficients.

In closing we remark that it is possible to further relax the assumptions of this global fit without losing a stable minimum. Namely, it is possible to leave also the Wilson coefficient $\left[C_{u \varphi}\right]_{22}$ as a free parameter in the fit. This is thanks to the direct measurement of the $h \rightarrow c \bar{c}$ signal strength at the LHC, which constrains the possible magnitude of charm Yukawa coupling modifications due to $\left[C_{u \varphi}\right]_{22}$. In the relaxed 32-parameter fit the results are mostly unchanged with respect to those displayed in table 2, except for a threefold increase of the error on the parameter $\delta c_{z}$. That increase happens because introducing $\left[C_{u \varphi}\right]_{22}$ opens an approximately flat direction corresponding to simultaneously increasing 


\begin{tabular}{|c|c|c|c|c|}
\hline Coeff. & central & & unc. & pull $[\sigma]$ \\
\hline$c_{W}$ & 0.053 & \pm & 1.8 & 0.0 \\
\hline$c_{\gamma \gamma}$ & -0.048 & \pm & 0.1 & 0.5 \\
\hline$c_{g g}$ & -0.017 & \pm & 0.014 & 1.2 \\
\hline$c_{z \square}$ & 4.4 & \pm & 6.0 & 0.7 \\
\hline$c_{z \gamma}$ & -0.58 & \pm & 0.61 & 0.9 \\
\hline$c_{z z}$ & -10.0 & \pm & 15.0 & 0.7 \\
\hline$\delta c_{z}$ & 0.44 & \pm & 1.5 & 0.3 \\
\hline$\delta g_{L 11}^{W l}$ & -0.083 & \pm & 0.052 & 1.6 \\
\hline$\delta g_{L 22}^{W l}$ & -0.23 & \pm & 0.082 & 2.8 \\
\hline$\delta g_{L 33}^{W l}$ & 0.27 & \pm & 0.099 & 2.8 \\
\hline$\delta g_{L 11}^{Z e}$ & -0.0056 & \pm & 0.0052 & 1.1 \\
\hline$\delta g_{L 22}^{Z e}$ & 0.00043 & \pm & 0.018 & 0.0 \\
\hline$\delta g_{L 33}^{Z e}$ & -0.0036 & \pm & 0.01 & 0.4 \\
\hline$\delta g_{L 11}^{Z d}$ & -0.072 & \pm & 0.61 & 0.1 \\
\hline$\delta g_{L 22}^{Z d}$ & 0.34 & \pm & 0.53 & 0.6 \\
\hline$\delta g_{L 33}^{Z d}$ & 0.054 & \pm & 0.027 & 2.0 \\
\hline$\delta g_{R 11}^{Z d}$ & 1.4 & \pm & 0.94 & 1.5 \\
\hline$\delta g_{R 22}^{Z d}$ & 0.29 & \pm & 0.72 & 0.4 \\
\hline$\delta g_{R 33}^{Z d}$ & 0.33 & \pm & 0.11 & 3.1 \\
\hline$\delta g_{R 11}^{Z e}$ & -0.0097 & \pm & 0.0055 & 1.8 \\
\hline$\delta g_{R 22}^{Z e}$ & -0.0025 & \pm & 0.021 & 0.1 \\
\hline$\delta g_{R 33}^{Z e}$ & 0.0074 & \pm & 0.01 & 0.7 \\
\hline$\delta g_{L 11}^{Z u}$ & 0.17 & \pm & 0.47 & 0.4 \\
\hline$\delta g_{L 22}^{Z u}$ & -0.032 & \pm & 0.072 & 0.5 \\
\hline$\delta g_{R 11}^{Z u}$ & 1.2 & \pm & 0.78 & 1.6 \\
\hline$\delta g_{R 22}^{Z u}$ & -0.055 & \pm & 0.084 & 0.7 \\
\hline$\left[C_{u \varphi}\right]_{33}$ & -0.28 & \pm & 2.7 & 0.1 \\
\hline$\left[C_{d \varphi}\right]_{33}$ & 0.018 & \pm & 0.049 & 0.4 \\
\hline$\left[C_{e \varphi}\right]_{22}$ & 0.004 & \pm & 0.0053 & 0.8 \\
\hline$\left[C_{e \varphi}\right]_{33}$ & -0.00059 & \pm & 0.018 & 0.0 \\
\hline$\left[C_{l l}\right]_{1221}$ & -0.64 & \pm & 0.2 & 3.2 \\
\hline
\end{tabular}

Table 2. Best-fit values (in units of $\mathrm{TeV}^{-2}$ ) and pulls of the Warsaw basis Wilson coefficient and their linear combinations defined in eq. (4.1) and eq. (4.3). 


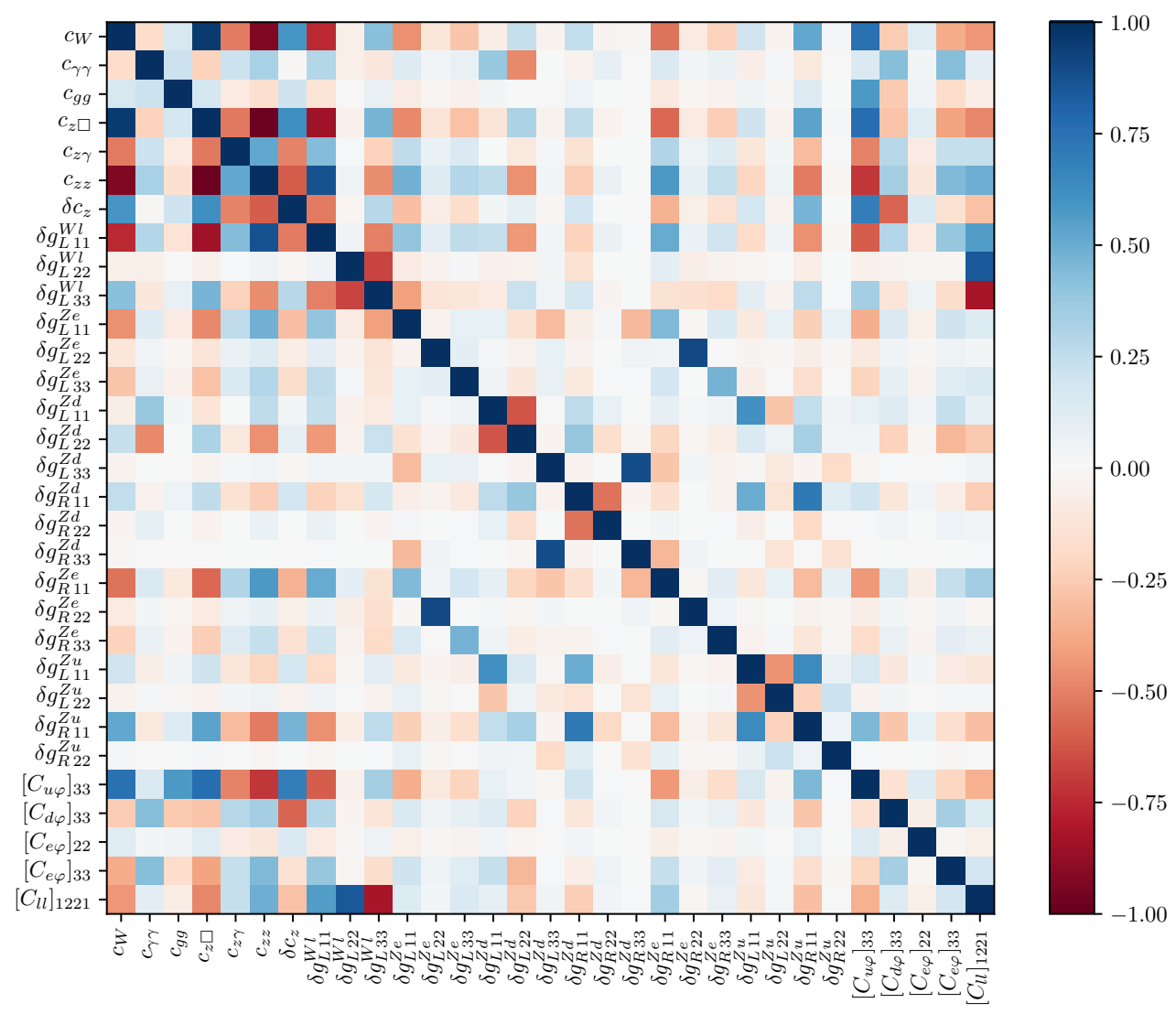

Figure 1. The correlation matrix for the constraints on Wilson coefficients in the 31-parameter fit in table 2.

both the total Higgs width and the $h V_{\mu} V_{\mu}$ couplings. The flat direction is lifted precisely thanks to the direct $h \rightarrow c \bar{c}$ measurement [23, 47].

\section{Applications}

\subsection{Oblique parameters}

Our first example application is the fit to oblique parameters starting from our general likelihood. To this end we assume that, at the energy scale $\mu \sim m_{Z}$, only two Wilson coefficients $C_{\varphi W B}$ and $C_{\varphi D}$ are non-negligible. We set all the remaining parameters to zero, and minimize the resulting two-dimensional likelihood. This procedure is directly related to the classic assumption that new physics enters via the so-called oblique $S$ and $T$ parameters [66, 67], with the identification

$$
C_{\varphi W B}=\frac{g_{L} g_{Y}}{16 \pi v^{2}} S, \quad C_{\varphi D}=-\frac{g_{L}^{2} g_{Y}^{2}}{2 \pi\left(g_{L}^{2}+g_{Y}^{2}\right) v^{2}} T .
$$

Obviously, this simple example is not using the full flavourful power of our approach. Nevertheless it is useful to present here in order to connect and compare to previous works. 


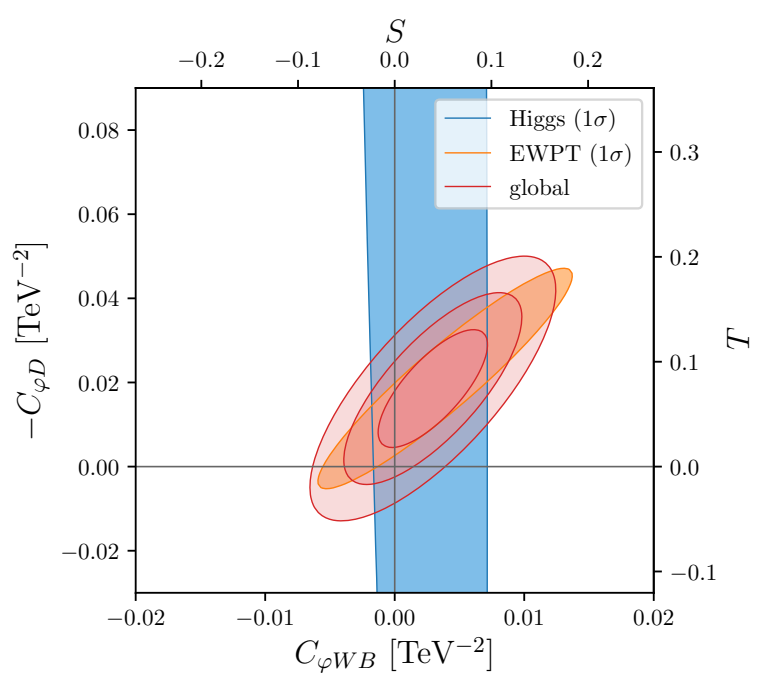

Figure 2. $S$ - $T$ fit using our combined Higgs and electroweak likelihood. We assume the only nonnegligible Wilson coefficients of dimension-6 operators at the scale $m_{Z}$ are $c_{\varphi D}$ and $c_{\varphi W B}$. This is equivalent to the usual fit to the oblique $S$ and $T$ parameters via the map in eq. (5.1). We show the $1 \sigma$ preferred region separately for Higgs (blue) and electroweak (orange) data. The red contours mark the $1 \sigma, 2 \sigma$, and $3 \sigma$ preferred regions using the combined likelihood.

For the Wilson coefficients at the scale $m_{Z}$ we find

$$
C_{\varphi W B}=0.0027 \pm 0.0028 \mathrm{TeV}^{-2}, \quad C_{\varphi D}=-0.0170 \pm 0.0094 \mathrm{TeV}^{-2}
$$

with the correlation coefficient $\rho=-0.74$. This translates to $S=0.035 \pm 0.038, T=$ $0.066 \pm 0.036$. The best fit ellipses are shown in figure 2 , for the combined likelihood, and for the electroweak and Higgs likelihoods separately. It can be seen that the LHC Higgs data contribute to constraining the $S$ parameter, mostly via measurements of the $h \rightarrow \gamma \gamma$ rate [14].

\subsection{Custodial vector resonance model}

Another example we consider is the model with an $\mathrm{SU}(2)$ triplet $V_{\mu}^{I}$ of massive vector resonances coupled to the SM Higgs, lepton $l$ and quark $q$ doublets as

$$
\mathcal{L} \supset \frac{1}{2} V_{\mu}^{I}\left(i g_{H} H^{\dagger} \tau^{I} D_{\mu} H-i g_{H} D_{\mu} H^{\dagger} \tau^{I} H+\sum_{i} g_{l_{i}} \bar{l}_{i} \tau^{I} \gamma^{\mu} l_{i}+\sum_{i} g_{q_{i}} \bar{q}_{i} \tau^{I} \gamma^{\mu} q_{i}\right)
$$

where $i=1,2,3$ is the SM generation index, and we allow the couplings to be flavour-nonuniversal. This kind of resonances and interactions arises e.g. in composite Higgs or warped extra-dimensional scenarios. The parameter space of our simplified model is characterized by 7 couplings $g_{k}$ and the resonance mass $M$. Assuming $\mathrm{U}(3)_{q} \times \mathrm{U}(3)_{l}$ flavour symmetry would reduce the number of independent couplings to three: $g_{H}, g_{l}$ and $g_{q}$. Integrating out the massive resonance leads to the SMEFT with the following Wilson coefficients of 
the operators in table 1:

$\left[C_{\varphi l}^{(3)}\right]_{i i}=-\frac{g_{H} g_{l_{i}}}{4 M^{2}}, \quad\left[C_{\varphi q}^{(3)}\right]_{i i}=-\frac{g_{H} g_{q_{i}}}{4 M^{2}}, \quad C_{\varphi \square}=-3 \frac{g_{H}^{2}}{8 M^{2}}, \quad\left[C_{f \varphi}\right]_{i i}=-\frac{g_{H}^{2} y_{f_{i}}}{4 M^{2}}, \quad\left[C_{l l}\right]_{1221}=-\frac{g_{l_{1}} g_{l_{2}}}{2 M^{2}}$.

where $y_{f_{i}}=\frac{\sqrt{2} m_{f_{i}}}{v}$ is the Yukawa coupling of the fermion $f_{i}, f=u, d, l$. As usual, only the ratios coupling/mass are available to a low-energy observer. Thus the SMEFT parameter space describing our simplified model is 7-dimensional in the generic case, and 3dimensional in the $\mathrm{U}(3)_{q} \times \mathrm{U}(3)_{l}$ limit. We ignore the effects of the operator $Q_{\varphi}$, which only affects double Higgs production and is very weakly constrained at present. Four-fermion operators other than $\left[Q_{l l}\right]_{1221}$, also generated in this model, are neglected in this analysis. They do not enter into the Higgs and electroweak observables at tree level, but they may affect other precision observables (e.g. LEP-2 fermion scattering, low-energy parity violation, Drell-Yan production at the LHC). Ignoring these effects is justified assuming $\left|g_{H}\right| \gg\left|g_{f_{i}}\right|$. Such coupling hierarchies can arise naturally in the composite/extra-dimensional scenario, e.g. via fermion localization in an extra dimension. We note that, in the limit $\left|g_{H}\right| \gg\left|g_{f_{i}}\right|$, constraints from the Higgs observables can be comparable or superior to those from the electroweak observables, which further motivates combining the two.

In this simplified model, one observes a profound difference between the allowed parameter space in the flavour-symmetric and generic cases. In the $\mathrm{U}(3)_{q} \times \mathrm{U}(3)_{l}$ case we find the $1 \sigma$ confidence intervals

$$
\frac{g_{H}}{M}=0.236_{-0.098}^{+0.086} \mathrm{TeV}^{-1}, \quad \frac{g_{l}}{g_{H}}=0.057 \pm 0.039, \quad \frac{g_{q}}{g_{H}}=0.014 \pm 0.040 .
$$

On the other hand, for generic flavour-dependent couplings the limits can be weaker:

$$
\begin{array}{llll}
\frac{g_{H}}{M}=0.231_{-0.107}^{+0.067} \mathrm{TeV}^{-1}, & \frac{g_{l_{1}}}{g_{H}}=0.054 \pm 0.040, & \frac{g_{l_{2}}}{g_{H}}=0.100 \pm 0.060, & \frac{g_{l_{3}}}{g_{H}}=0.009 \pm 0.068 \\
\frac{g_{q_{1}}}{g_{H}}=-0.05 \pm 0.25, & \frac{g_{q_{2}}}{g_{H}}=0.11 \pm 0.27, & \frac{g_{q_{3}}}{g_{H}}=-0.027 \pm 0.094 .
\end{array}
$$

We see the for the resonance coupling to the Higgs field the limits are fairly independent on how the resonance couples to the SM fermions. This is because the limit is dominated by the Higgs data. On the other hand, the limits on the resonance couplings to the SM fermions are sensitive to whether or not $\mathrm{U}(3)_{q} \times \mathrm{U}(3)_{l}$ is assumed, as also illustrated in figure 3. The difference is most dramatic for the couplings to the first two generation of quarks, which are allowed to be an order of magnitude larger in the generic case than in the $\mathrm{U}(3)_{q} \times \mathrm{U}(3)_{l}$ case.

\section{Summary}

In this paper we presented an updated fit of the dimension-6 SMEFT operators to combined Higgs and electroweak precision data. We included the most recent ATLAS and CMS combinations of run-2 Higgs data, and also complemented the LEP electroweak data with a couple of recent measurements in hadronic machines. The analysis is based on an open 


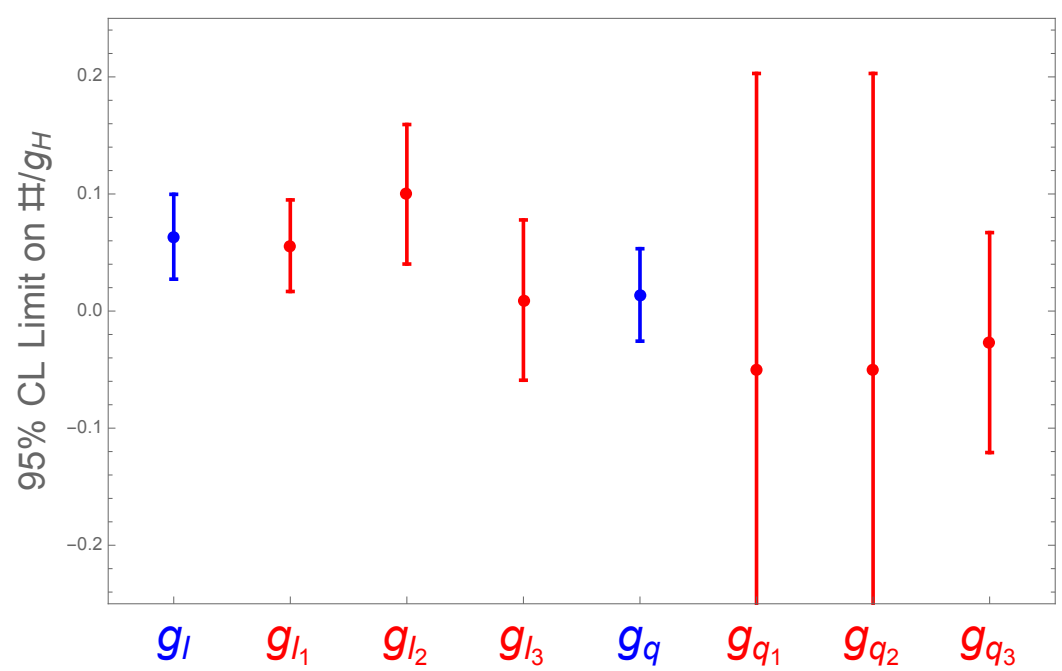

Figure 3. Comparison of the limits on the parameter space of the $\mathrm{SU}(2)$ vector resonance model in the $\mathrm{U}(3)_{q} \times \mathrm{U}(3)_{l}$ flavour universal (blue) and flavour generic (red) cases. We display the $95 \% \mathrm{CL}$ confidence intervals for the resonance couplings to the SM fermions $g_{f_{i}}$ normalized to its coupling to the Higgs $g_{H}$, see eq. (5.3). In the flavour universal case we have $g_{f_{i}}=g_{f}$ for all three generations, in which case the bounds are much stronger than in the flavour generic case when $g_{f_{1}} \ldots g_{f_{3}}$ are all independent. For the coupling $g_{H}$, the bounds are dominated by the LHC Higgs data and thus weakly depend on the flavour assumptions. We find the $95 \% \mathrm{CL}$ limits: $\left|g_{H}\right| \leq 0.97 \mathrm{TeV}^{-1}$ in the flavour-universal case and $\left|g_{H}\right| \leq 0.82 \mathrm{TeV}^{-1}$ in the case of generic flavour-dependent couplings.

source Python code, which allows the users to easily to scrutinize, reproduce, or update our analysis. The code and conventions follow the WCxf format, so that our results can easily be imported by other analysis programs.

At the physics level, the main improvement compared to earlier works is that we allow for a completely general flavour structure of dimension-6 operators. Thanks to that, the likelihood we provide can be used to constrain a broad class of BSM models beyond the $\mathrm{U}(3)^{5}$ or minimal flavour violation paradigm.

\section{Acknowledgments}

AF is partially supported by the European Union's Horizon 2020 research and innovation programme under the Marie Skłodowska-Curie grant agreements No. 690575 and No. 674896. We thank Ken Mimasu, Michael Spannowsky, and Michael Trott for useful discussions. This research was supported by the Munich Institute for Astro- and Particle Physics (MIAPP) of the DFG Excellence Cluster Origins (https://www.originscluster.de/).

Open Access. This article is distributed under the terms of the Creative Commons Attribution License (CC-BY 4.0), which permits any use, distribution and reproduction in any medium, provided the original author(s) and source are credited. 


\section{References}

[1] ATLAS collaboration, Observation of a new particle in the search for the Standard Model Higgs boson with the ATLAS detector at the LHC, Phys. Lett. B 716 (2012) 1 [arXiv: 1207.7214] [INSPIRE].

[2] CMS collaboration, Observation of a New Boson at a Mass of $125 \mathrm{GeV}$ with the CMS Experiment at the LHC, Phys. Lett. B 716 (2012) 30 [arXiv:1207.7235] [InSPIRE].

[3] D. Carmi, A. Falkowski, E. Kuflik and T. Volansky, Interpreting LHC Higgs Results from Natural New Physics Perspective, JHEP 07 (2012) 136 [arXiv:1202.3144] [INSPIRE].

[4] A. Azatov, R. Contino and J. Galloway, Model-Independent Bounds on a Light Higgs, JHEP 04 (2012) 127 [Erratum JHEP 04 (2013) 140] [arXiv: 1202.3415] [INSPIRE].

[5] J.R. Espinosa, C. Grojean, M. Muhlleitner and M. Trott, Fingerprinting Higgs Suspects at the LHC, JHEP 05 (2012) 097 [arXiv: 1202.3697] [INSPIRE].

[6] T. Corbett, O.J.P. Eboli, J. Gonzalez-Fraile and M.C. Gonzalez-Garcia, Robust Determination of the Higgs Couplings: Power to the Data, Phys. Rev. D 87 (2013) 015022 [arXiv: 1211.4580] [INSPIRE].

[7] A. Pomarol and F. Riva, Towards the Ultimate SM Fit to Close in on Higgs Physics, JHEP 01 (2014) 151 [arXiv:1308.2803] [INSPIRE].

[8] B. Dumont, S. Fichet and G. von Gersdorff, A Bayesian view of the Higgs sector with higher dimensional operators, JHEP 07 (2013) 065 [arXiv: 1304.3369] [INSPIRE].

[9] T. Corbett, O.J.P. Éboli, J. Gonzalez-Fraile and M.C. Gonzalez-Garcia, Determining Triple Gauge Boson Couplings from Higgs Data, Phys. Rev. Lett. 111 (2013) 011801 [arXiv: 1304.1151] [INSPIRE].

[10] J. Ellis, V. Sanz and T. You, The Effective Standard Model after LHC Run I, JHEP 03 (2015) 157 [arXiv:1410.7703] [InSPIRE].

[11] A. Falkowski, M. González-Alonso, A. Greljo and D. Marzocca, Global constraints on anomalous triple gauge couplings in effective field theory approach, Phys. Rev. Lett. 116 (2016) 011801 [arXiv:1508.00581] [INSPIRE].

[12] A. Butter, O.J.P. Éboli, J. Gonzalez-Fraile, M.C. Gonzalez-Garcia, T. Plehn and M. Rauch, The Gauge-Higgs Legacy of the LHC Run I, JHEP 07 (2016) 152 [arXiv:1604.03105] [INSPIRE].

[13] L. Berthier, M. Bjørn and M. Trott, Incorporating doubly resonant $W^{ \pm}$data in a global fit of SMEFT parameters to lift flat directions, JHEP 09 (2016) 157 [arXiv:1606.06693] [INSPIRE].

[14] J. Ellis, C.W. Murphy, V. Sanz and T. You, Updated Global SMEFT Fit to Higgs, Diboson and Electroweak Data, JHEP 06 (2018) 146 [arXiv: 1803.03252] [INSPIRE].

[15] A. Biekötter, T. Corbett and T. Plehn, The Gauge-Higgs Legacy of the LHC Run II, SciPost Phys. 6 (2019) 064 [arXiv:1812.07587] [INSPIRE].

[16] J. De Blas et al., HEPfit: a Code for the Combination of Indirect and Direct Constraints on High Energy Physics Models, arXiv:1910.14012 [INSPIRE].

[17] J. de Blas, J.C. Criado, M. Pérez-Victoria and J. Santiago, Effective description of general extensions of the Standard Model: the complete tree-level dictionary, JHEP 03 (2018) 109 [arXiv: 1711.10391] [INSPIRE]. 
[18] LHCb collaboration, Search for lepton-universality violation in $B^{+} \rightarrow K^{+} \ell^{+} \ell^{-}$decays, Phys. Rev. Lett. 122 (2019) 191801 [arXiv: 1903.09252] [INSPIRE].

[19] LHCb collaboration, Test of lepton universality with $B^{0} \rightarrow K^{* 0} \ell^{+} \ell^{-}$decays, JHEP 08 (2017) 055 [arXiv: 1705.05802] [INSPIRE].

[20] HFLAV collaboration, Averages of b-hadron, c-hadron and $\tau$-lepton properties as of summer 2016, Eur. Phys. J. C 77 (2017) 895 [arXiv:1612.07233] [InSPIRE].

[21] J. Aebischer et al., WCxf: an exchange format for Wilson coefficients beyond the Standard Model, Comput. Phys. Commun. 232 (2018) 71 [arXiv:1712.05298] [INSPIRE].

[22] CMS collaboration, Combined measurements of Higgs boson couplings in proton-proton collisions at $\sqrt{s}=13 \mathrm{TeV}$, Eur. Phys. J. C 79 (2019) 421 [arXiv:1809.10733] [InSPIRE].

[23] ATLAS collaboration, Search for the Decay of the Higgs Boson to Charm Quarks with the ATLAS Experiment, Phys. Rev. Lett. 120 (2018) 211802 [arXiv:1802.04329] [INSPIRE].

[24] M. González-Alonso and J. Martin Camalich, Global Effective-Field-Theory analysis of New-Physics effects in (semi)leptonic kaon decays, JHEP 12 (2016) 052 [arXiv:1605.07114] [INSPIRE].

[25] A. Falkowski, M. González-Alonso and K. Mimouni, Compilation of low-energy constraints on 4-fermion operators in the SMEFT, JHEP 08 (2017) 123 [arXiv:1706.03783] [INSPIRE].

[26] J. Aebischer, J. Kumar, P. Stangl and D.M. Straub, A Global Likelihood for Precision Constraints and Flavour Anomalies, Eur. Phys. J. C 79 (2019) 509 [arXiv:1810.07698] [INSPIRE].

[27] D.M. Straub, flavio: a Python package for flavour and precision phenomenology in the Standard Model and beyond, arXiv:1810.08132 [INSPIRE].

[28] J. Aebischer, J. Kumar and D.M. Straub, Wilson: a Python package for the running and matching of Wilson coefficients above and below the electroweak scale, Eur. Phys. J. C 78 (2018) 1026 [arXiv: 1804.05033] [INSPIRE].

[29] W. Buchmüller and D. Wyler, Effective Lagrangian Analysis of New Interactions and Flavor Conservation, Nucl. Phys. B 268 (1986) 621 [InSPIRE].

[30] B. Grzadkowski, M. Iskrzynski, M. Misiak and J. Rosiek, Dimension-Six Terms in the Standard Model Lagrangian, JHEP 10 (2010) 085 [arXiv: 1008.4884] [INSPIRE].

[31] A. Efrati, A. Falkowski and Y. Soreq, Electroweak constraints on flavorful effective theories, JHEP 07 (2015) 018 [arXiv: 1503.07872] [INSPIRE].

[32] Particle Data Group, Review of Particle Physics, Phys. Rev. D 98 (2018) 030001 [INSPIRE].

[33] ATLAS collaboration, Measurement of the $W$-boson mass in pp collisions at $\sqrt{s}=7 \mathrm{TeV}$ with the ATLAS detector, Eur. Phys. J. C 78 (2018) 110 [Erratum ibid. C 78 (2018) 898] [arXiv: 1701.07240] [INSPIRE].

[34] D0 collaboration, A measurement of the $W \rightarrow \tau \nu$ production cross section in $p \bar{p}$ collisions at $\sqrt{s}=1.8$ TeV, Phys. Rev. Lett. 84 (2000) 5710 [hep-ex/9912065] [InSPIRE].

[35] LHCb collaboration, Measurement of forward $W \rightarrow e \nu$ production in pp collisions at $\sqrt{s}=8 \mathrm{TeV}$, JHEP 10 (2016) 030 [arXiv:1608.01484] [INSPIRE].

[36] P. Janot and S. Jadach, Improved Bhabha cross section at LEP and the number of light neutrino species, Phys. Lett. B 803 (2020) 135319 [arXiv:1912.02067] [INSPIRE]. 
[37] CMS collaboration, Measurement of the t-channel single-top-quark production cross section and of the $\left|V_{t b}\right|$ CKM matrix element in pp collisions at $\sqrt{s}=8 \mathrm{TeV}$, JHEP 06 (2014) 090 [arXiv: 1403.7366] [INSPIRE].

[38] ALEP, DELPHI, L3, OPAL and LEP ELECTROWEAK collaborations, Electroweak Measurements in Electron-Positron Collisions at W-Boson-Pair Energies at LEP, Phys. Rept. 532 (2013) 119 [arXiv:1302.3415] [INSPIRE].

[39] A. Falkowski and F. Riva, Model-independent precision constraints on dimension-6 operators, JHEP 02 (2015) 039 [arXiv: 1411.0669] [INSPIRE].

[40] ATLAS and CMS collaborations, Measurements of the Higgs boson production and decay rates and constraints on its couplings from a combined ATLAS and CMS analysis of the LHC pp collision data at $\sqrt{s}=7$ and $8 \mathrm{TeV}$, JHEP 08 (2016) 045 [arXiv: 1606.02266] [INSPIRE].

[41] ATLAS collaboration, Measurements of the Higgs boson production and decay rates and coupling strengths using pp collision data at $\sqrt{s}=7$ and 8 TeV in the ATLAS experiment, Eur. Phys. J. C 76 (2016) 6 [arXiv:1507.04548] [InSPIRE].

[42] CMS collaboration, Search for a Higgs Boson Decaying into a $Z$ and a Photon in pp Collisions at $\sqrt{s}=7$ and 8 TeV, Phys. Lett. B 726 (2013) 587 [arXiv:1307.5515] [INSPIRE].

[43] ATLAS collaboration, Combined measurements of Higgs boson production and decay using up to $80 \mathrm{fb}^{-1}$ of proton-proton collision data at $\sqrt{\mathrm{s}}=13$ TeV collected with the ATLAS experiment, Phys. Rev. D 101 (2020) 012002 [arXiv:1909.02845] [INSPIRE].

[44] ATLAS collaboration, Search for the dimuon decay of the Higgs boson in pp collisions at $\sqrt{s}=13$ TeV with the ATLAS detector, Phys. Rev. Lett. 119 (2017) 051802 [arXiv: 1705.04582] [INSPIRE].

[45] ATLAS collaboration, Searches for the $Z \gamma$ decay mode of the Higgs boson and for new high-mass resonances in pp collisions at $\sqrt{s}=13$ TeV with the ATLAS detector, JHEP 10 (2017) 112 [arXiv:1708.00212] [INSPIRE].

[46] CMS collaboration, Search for the decay of a Higgs boson in the $\ell \ell \gamma$ channel in proton-proton collisions at $\sqrt{s}=13 \mathrm{TeV}$, JHEP 11 (2018) 152 [arXiv:1806.05996] [INSPIRE].

[47] CMS collaboration, Search for the standard model Higgs boson decaying to charm quarks, CMS-PAS-HIG-18-031 (2019) [INSPIRE].

[48] I. Brivio, Y. Jiang and M. Trott, The SMEFTsim package, theory and tools, JHEP 12 (2017) 070 [arXiv: 1709.06492] [INSPIRE].

[49] J. Alwall et al., The automated computation of tree-level and next-to-leading order differential cross sections and their matching to parton shower simulations, JHEP 07 (2014) 079 [arXiv: 1405.0301] [INSPIRE].

[50] R. Alonso, E.E. Jenkins, A.V. Manohar and M. Trott, Renormalization Group Evolution of the Standard Model Dimension Six Operators III: Gauge Coupling Dependence and Phenomenology, JHEP 04 (2014) 159 [arXiv: 1312.2014] [INSPIRE].

[51] S. Dawson and P.P. Giardino, Electroweak and QCD corrections to $Z$ and $W$ pole observables in the standard model EFT, Phys. Rev. D 101 (2020) 013001 [arXiv: 1909.02000] [INSPIRE].

[52] S. Dawson and P.P. Giardino, Electroweak corrections to Higgs boson decays to $\gamma \gamma$ and $W^{+} W^{-}$in standard model EFT, Phys. Rev. D 98 (2018) 095005 [arXiv: 1807.11504] [INSPIRE]. 
[53] A. Dedes, M. Paraskevas, J. Rosiek, K. Suxho and L. Trifyllis, The decay $h \rightarrow \gamma \gamma$ in the Standard-Model Effective Field Theory, JHEP 08 (2018) 103 [arXiv:1805.00302] [INSPIRE].

[54] S. Dawson and P.P. Giardino, Higgs decays to $Z Z$ and $Z \gamma$ in the standard model effective field theory: An NLO analysis, Phys. Rev. D 97 (2018) 093003 [arXiv:1801.01136] [INSPIRE].

[55] C. Hartmann and M. Trott, Higgs Decay to Two Photons at One Loop in the Standard Model Effective Field Theory, Phys. Rev. Lett. 115 (2015) 191801 [arXiv:1507.03568] [InSPIRE].

[56] Z. Zhang, Time to Go Beyond Triple-Gauge-Boson-Coupling Interpretation of W Pair Production, Phys. Rev. Lett. 118 (2017) 011803 [arXiv:1610.01618] [INSPIRE].

[57] S. Banerjee, C. Englert, R.S. Gupta and M. Spannowsky, Probing Electroweak Precision Physics via boosted Higgs-strahlung at the LHC, Phys. Rev. D 98 (2018) 095012 [arXiv: 1807.01796] [INSPIRE].

[58] Y. Chen and R. Vega-Morales, Extracting Effective Higgs Couplings in the Golden Channel, JHEP 04 (2014) 057 [arXiv: 1310.2893] [INSPIRE].

[59] A. Falkowski, Effective field theory approach to LHC Higgs data, Pramana 87 (2016) 39 [arXiv: 1505.00046] [INSPIRE].

[60] I. Brivio, T. Corbett and M. Trott, The Higgs width in the SMEFT, JHEP 10 (2019) 056 [arXiv: 1906.06949] [INSPIRE].

[61] R.S. Gupta, A. Pomarol and F. Riva, BSM Primary Effects, Phys. Rev. D 91 (2015) 035001 [arXiv: 1405.0181] [INSPIRE].

[62] F. Krauss, S. Kuttimalai and T. Plehn, LHC multijet events as a probe for anomalous dimension-six gluon interactions, Phys. Rev. D 95 (2017) 035024 [arXiv:1611.00767] [INSPIRE].

[63] T. Corbett, O.J.P. Eboli, D. Goncalves, J. Gonzalez-Fraile, T. Plehn and M. Rauch, The Higgs Legacy of the LHC Run I, JHEP 08 (2015) 156 [arXiv:1505.05516] [InSPIRE].

[64] C. Englert, R. Kogler, H. Schulz and M. Spannowsky, Higgs coupling measurements at the LHC, Eur. Phys. J. C 76 (2016) 393 [arXiv:1511.05170] [INSPIRE].

[65] M. Grazzini, A. Ilnicka, M. Spira and M. Wiesemann, Modeling BSM effects on the Higgs transverse-momentum spectrum in an EFT approach, JHEP 03 (2017) 115 [arXiv: 1612.00283] [INSPIRE].

[66] M.E. Peskin and T. Takeuchi, Estimation of oblique electroweak corrections, Phys. Rev. D 46 (1992) 381 [INSPIRE].

[67] J.D. Wells and Z. Zhang, Effective theories of universal theories, JHEP 01 (2016) 123 [arXiv: 1510.08462] [INSPIRE]. 